\title{
Research submission categories
}

\author{
I. Diane Cooper, AHIP \\ DOI: http://dx.doi.org/10.3163/1536-5050.104.2.002
}

The Journal of the Medical Library Association's (JMLA's) research submission categories have four subsections. Articles are positioned in subsections according to the strength of their research evidence.

The strength of research evidence depends on the quality, quantity, and type of studies conducted. Levels of research evidence are a continuum. This is as true for library-related research as it is for clinical science, social science, and other disciplines. It is important to remind both makers of research results and users of research results of this fact.

Systematic reviews of high-quality experimental studies provide the strongest evidence (both positive and negative) for research users in their library practice decisions. Next to them, controlled trials and cohort studies can provide strong evidence.

Descriptive surveys and qualitative studies can provide important initial information. They can provide insights, stimulate discussion, and serve as a basis for generating outcome studies. The weakest evidence comes from expert opinions and commentaries.

More than fifteen years ago, Jonathan Eldredge, AHIP, outlined levels for evidence-based librarianship (EBL) research and offered standards to be integrated into the health sciences librarian culture [1]. Evidence levels of EBL research ranged from high to low are: systematic reviews, randomized control trials, controlled-comparison studies, cohort studies, decision analysis, and qualitative research that includes focus groups, ethnographic observations, and historical analysis.

To support the EBL process and make it clearer both for those who conduct research and for those who read research, the JMLA research submission categories now comprise the following four research category labels. These are essentially the same as the previous JMLA categories, except they are aligned more closely with the concept of levels of evidence.

Please note that these levels apply to the strength of the evidence, not the quality of the research. People often confuse strength of evidence with quality of research. Very high-quality research can occur at each level.

\section{PAPERS}

- Randomized control trials

- Controlled comparison studies, when groups are representative

- Cohort studies, which typically follow two groups (cohorts), when one group has been exposed to a variable such as a situation, event, teaching, and so on

- Systematic reviews, where there is a comprehensive, organized review evaluating a body of literature on a specific topic

\section{SURVEYS AND STUDIES}

- Descriptive surveys

- Intervention studies in which there are no control groups

- Intervention studies with low power

- Qualitative research studies that follow wellconstructed and rigorous methodology, are

representative, and provide insight about major issues

- Studies with non-generalizable results when they provoke important discussion

\section{RESEARCH COMMUNICATIONS}

- Preliminary findings

- Feasibility studies

- Tests of concept

\section{CASE STUDIES}

- Description of a new or innovative resolution to a problem or issue

\section{COMMENT}

These labels can help readers use research to make evidence-based decisions. They also contribute to rational space utilization of scholarly articles in the 
$J M L A$. These labels do not refer to quality of research; quality of research depends on the design and execution of the research. The length of a research article likewise does not measure quality of research. Very excellent research often can be presented in shorter articles.

These evidence levels are traditionally used for quantitative studies, but the concepts also apply to qualitative research. To paraphrase experts from another profession, librarians "often approach situations from a perspective that is comprehensive and holistic, rather than reductionistic and deterministic" [2]. All quality health sciences libraryrelated research is welcome in the JMLA.

\section{REFERENCES}

1. Eldredge JD. Evidence-based librarianship: an overview. Bull Med Libr Assoc. 2000 Oct;88(4):289-302. 2. Cesurio S, Morin K, Santa-Donato A. Evaluating the level of evidence of qualitative research. J Obstet Gynecol Neonatal Nurs. 2002 Nov-Dec;31(6):708-14.

\section{AUTHOR'S AFFILIATION}

I. Diane Cooper, AHIP, jmlaeditorbox@gmail.com, Editor-in-Chief, Journal of the Medical Library Association

\section{CORRECTIONS}

\section{VOLUME 104}

\section{4(1) January, page 87}

Duncan S. Health sciences librarianship [review]. J Med Libr Assoc. 2016 Jan;104(1):86-7. http://dx.doi. org/10.3163/1536-5050.104.1.018.

The correct title of chapter eleven of Health Sciences Librarianship is "Instruction in Health Sciences Libraries."

DOI: http://dx.doi.org/10.3163/1536-5050.104.2.025

\section{4(1) January, page E1}

Mitchell N. Proceedings, 115th Annual Meeting, Medical Library Association, Inc. Austin, TX. J Med Libr Assoc. 2016 Jan;104(1):E1-E33.

The correct digital object identifier for the proceedings of the 115th annual meeting is http://dx.doi.org/ 10.3163/1536-5050.104.1.E1. The JMLA regrets the error.

DOI: http://dx.doi.org/10.3163/1536-5050.104.2.026 